\title{
ANNIHILATION OF o-POSITRONIUM IN THE VICINITY OF THE CRITICAL MIXING POINT
}

\author{
K. Jerie ${ }^{a}$, A. Baranowski ${ }^{a}$, J. Glinski ${ }^{b}$ AND K. ORzechowski ${ }^{b}$ \\ ${ }^{a}$ Institute of Experimental Physics, University of Wrocław \\ Pl. Maxa Borna 9, 50-204 Wrocław, Poland \\ ${ }^{b}$ Faculty of Chemistry, University of Wroctaw \\ F. Joliot-Curie 14, 50-383 Wrocław, Poland
}

\begin{abstract}
Positron annihilation spectra were investigated in the critical region close to the consolute point of ethanol + dodecane mixture. The anomalies of the positron lifetimes and intensities were small, nevertheless it was found that the intensity of short-living components of annihilation spectrum seems to increase close to the critical temperature.
\end{abstract}

PACS numbers: $05.70 . \mathrm{Jk}, 78.70 . \mathrm{Bj}$

\section{Introduction}

Let us consider a liquid mixture of two-component mutually soluble in high temperatures and forming saturated phases in low ones. At the temperature and the concentration of maximum of the coexistence curve, the phase transition is classified as continuous and belongs to $(3,1)$ universality class. The critical anomaly of second derivatives of the Gibbs free energy (heat capacity, isothermal compressibility, osmotic compressibility, etc.) results in large and long-living concentration fluctuations impeding most of macroscopic properties of such binary mixtures. Some of the quantities, as light scattering [1-5], non-linear dielectric effect [6-8], absorption of ultrasonic waves [9-11] exhibit strong critical anomalies while others, as density [12] or electric permittivity [13, 14] - weak ones. Many critical properties could be qualitatively predicted when regarding a mixture in the vicinity of critical point as an inhomogeneous one. This attempt was successively used in understanding of critical phenomena of low frequency electric permittivity and conductivity [15-20], as well as in nonlinear dielectric effect [21]. It has to be pointed out that formal similarities between critical mixtures and real inhomogeneous mixtures (for example emulsions) are true only very close to the critical point, where concentration fluctuations are long-range and long-living.

Positron annihilation measurements are a suitable experimental technique for investigation of solid minerals [22], polymers [23], as well as for investigation of clathrate-like structures in diluted aqueous mixtures of non-electrolytes [24-26]. 
The annihilation experiments involve the measurement of $\gamma$ radiation formed during annihilation of positrons emitted by a radioactive source $\left({ }^{22} \mathrm{Na}\right)$. Positrons could annihilate directly or form para- or ortho-positronium and then annihilate. In liquids, the time spectrum is usually resolved into three components. The short components $\left(\tau_{1}, \tau_{2}, I_{1}, I_{2}\right.$, where $\tau_{i}$ and $I_{i}$ are lifetime and intensity, respectively) are associated with direct annihilation and that of para-positronium. The lifetime of ortho-positronium $\left(\tau_{3}\right)$ is a few times larger than $\tau_{1}$ and $\tau_{2}$. In order to ortho-positronium could exist, sufficiently large cavities in the investigated medium have to be obtainable. The possibility of the formation of these cavities depends on the structure of liquid and, especially, on intermolecular interactions. This is the basis of applying positron annihilation measurements in structural problems in liquids.

In our previous paper devoted to investigation of positron annihilation in a critical methanol + cyclohexane mixture [27] we found a decrease in lifetime of ortho-positronium $\left(\tau_{3}\right)$ when temperature was approaching the critical one. It was assumed that the ortho-positronium lifetime anomaly was a result of critical slowing down or a selective capturing of it in those areas (fluctuations) where positroniums live shorter.

In this paper we present the results obtained in an ethanol + dodecane critical mixture. The critical temperature of this mixture has a very convenient value $\left(T_{\mathrm{c}}=12.5^{\circ} \mathrm{C}\right)$ which allows us to provide a more precise temperature stabilization and to verify previously derived conclusions more confidently.

\section{Experimental}

The mean lifetimes of positrons were measured with a standard system [28-30] based on the "fast-slow" coincidence technique. The radioactive source was surrounded by the investigated mixture. Because positron annihilation experiments are very time consuming (approximately $12 \mathrm{~h}$ per one temperature point) the special attempt has to be made for careful temperature stabilization. In our experiment the temperature stabilization was provided by a Peltier thermostat (ECOLAB, Poland) and the temperature drift in a course of a single experiment was not larger than $0.1 \mathrm{~K}$. Temperature was measured by means of calibrated thermistor with resolution of $\pm 0.01 \mathrm{~K}$. Ethanol was prepared using the material obtained after azeotropic distillation with benzene. Alcohol was dried using freshly ignited calcium oxide and then by magnesium activated by iodine and finally distilled. Dodecane was distilled under reduced pressure over metallic sodium. The mixture of close critical concentration (mole fraction of alcohol $x=0.67$ [8]) was prepared by weighing. Location of the critical point was verified using equal volumes criterion.

\section{Results and discussion}

In the vicinity of critical mixing point, a mixture could be $\cdot \cdots$ mally regarded as an inhomogeneous one because of large and long-living concentration fluctuations. Nonlinear dielectric properties investigated already in the same mixture [8] demonstrate strong anomalies caused by large concentration fluctuations in the 
critical region. Now, in a similar temperature range $\left(25^{\circ} \mathrm{C}\right.$ to $\left.10^{\circ} \mathrm{C}\right)$, the positron annihilation experiments were performed to prove whether microinhomogeneity produced by critical fluctuations influences the annihilation process. Temperature was decreasing stepwise in $1 \mathrm{~K}$ intervals when fär from the critical temperature, and approximately in $0.2 \mathrm{~K}$ intervals in the vicinity of $T_{\mathrm{c}}$. The spectra of $\gamma$ radiation resulting from direct annihilation, annihilation of ortho- as well as of para-positronium were numerically resolved into three components. The temperature dependence of lifetimes $\left(\tau_{1}, \tau_{2}, \tau_{3}\right)$ and intensities $\left(I_{1}+I_{2}, I_{3}\right)$ are presented in Figs. 1 and 2, respectively and compared to the previously obtained nonlinear dielectric effect data [8].
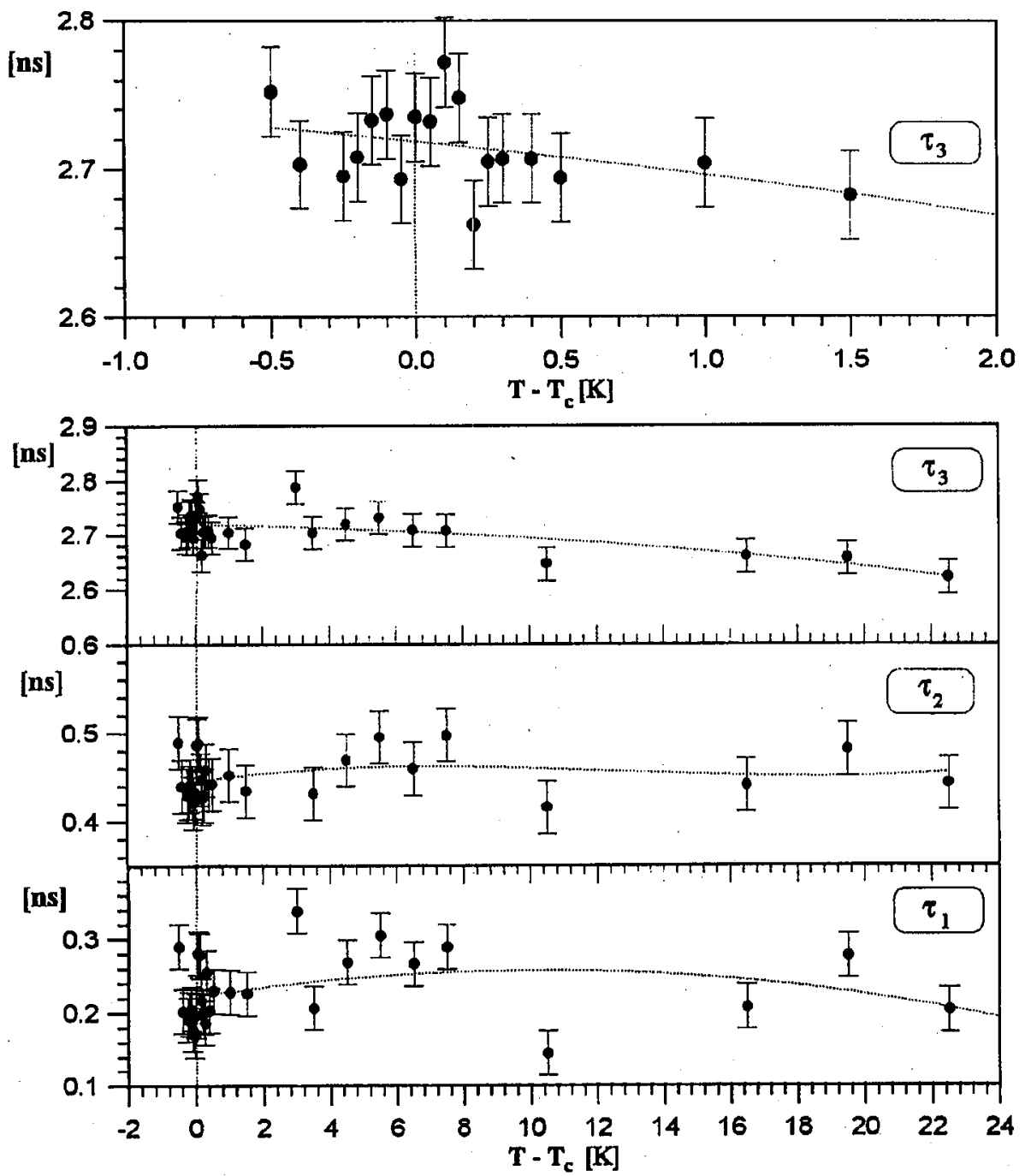

Fig. 1. The temperature dependences of positron lifetimes in ethanol + dodecane critical mixture. 

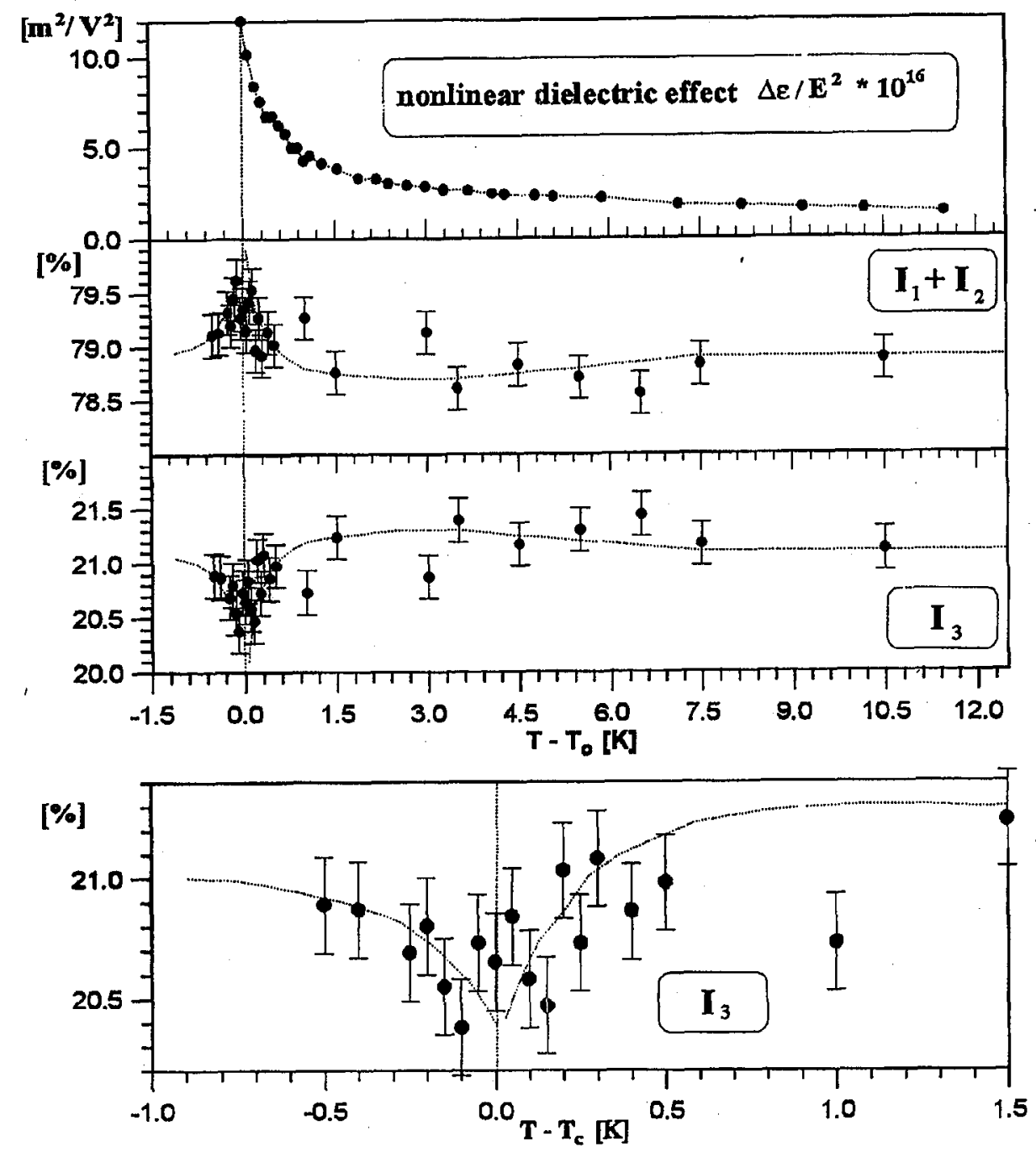

Fig. 2. The temperature dependences of the intensities of short-living components $\left(I_{1}+I_{2}\right)$ and long-living one $\left(I_{3}\right)$, in ethanol + dodecane critical mixture compared with nonlinear dielectric effect data [8].

According to Fig. 1, a pronounced anomaly of nonlinear dielectric effect was found in the vicinity of $T_{\mathrm{c}}$ [8]. In the annihilation experiment, in spite of relatively high spreading of experimental points, at a similar temperature range a small increase in $I_{1}+I_{2}$ (and, consequently, a decrease in $I_{3}=100 \%-I_{1}-I_{2}$ ) is observed. The observed changes in the lifetimes of positrons (Fig. 2) do not allow us to speculate on the influence of critical fluctuations on this quantity, whereas in the previously investigated methanol + cyclohexane mixture, the lifetime of ortho-positronium $\left(\tau_{3}\right)$ seems to decrease close to critical temperature [27]. 
Is the increase in $I_{1}+I_{2}$ (and consequently the decrease in $I_{3}$ ), observed in the vicinity of the critical temperature, a result of local concentration fluctuations? Close to the critical temperature, fluctuations become long-range and long-living and, finally, when temperature is lower than the upper critical one, the mixture separates into two mutually saturated liquid phases. Just before the critical temperature, a mixture could be formally regarded as microscopically inhomogeneous: The previously observed anomalies in nonlinear dielectric effect allow us to localise the temperature range where fluctuations affect considerably properties of the investigated mixture. An increase in intensities of $I_{1}+I_{2}$ means that probability of the annihilation process determining the short living components increases. When passing through a mixture with large fluctuations, positrons emitted by the radioactive source should be preferentially annihilated in those regions (fluctuations) of the mixture where their lifetime is shorter (for instance because of larger probability of direct annihilation). Following this concept, it seems that the critical anomaly of positron annihilation spectrum should be more pronounced when the difference in electron densities of the components of a mixture increases. A mixture selected according to this property would give more evident anomalies in the vicinity of the critical mixing point and will be the subject of our future investigations.

\section{Acknowledgments}

This work was supported by the Committee for Scientific Research grant No. 3 T09A 05211 and by the University of Wrocław grant No. 2016/IFD/98.

\section{References}

[1] B. Beysens, A. Bourgou, H. Charlin, Phys. Lett. A 53, 236 (1975).

[2] L.A. Reith, H.L. Swinney, Phys. Rev. A 12, 1094 (1975).

[3] Y. Garrabos, G. Zalczer, D. Beysens, Phys. Rev. A 25, 1147 (1982).

[4] D.T. Jacobs, Phys. Rev. A 33, 2605 (1986).

[5] J.P. Rakotoniaina, L. Belkoura, D. Woermann, Z. Phys. Chem. 196, 237 (1996).

[6] J. Chrapeć, S.J. Rzoska, J. Zioło, Chem. Phys. 111, 115 (1987).

[7] J. Chrapeć, S.J. Rzoska, J. Zioło, Chem. Phys. 112, 471 (1988).

[8] K. Orzechowski, Physica B 172, 339 (1991).

[9] H. Tanaka, Y. Wada, H. Nakajima, Chem. Phys. 68, 223 (1982).

[10] G. Jaschull, H. Dunker, D. Woermann, Ber. Bunsenges. Phys. Chem. 88, 630 (1984).

[11] S.J. Fast, S.S. Yun, J. Chem. Phys. 83, 5888 (1985).

[12] P. Garsen, D. Woermann, Ber. Bunsenges. Phys. Chem. 88, 626 (1984).

[13] M. Konecki, Chem. Phys. Lett. 57, 90 (1978).

[14] K. Orzechowski, Ber. Bunsenges. Phys. Chem. 92, 931 (1988).

[15] J. Thoen, R. Kindt, W. Van Dael, M. Merabet, T.K. Bose, Physica A 156, 92 (1989).

[16] J. Hamelin, T.K. Bose, J. Thoen, Phys. Rev. A 42, 4735 (1990). 
[17] K. Orzechowski, J. Chem. Soc. Faraday Trans. 90, 2757 (1994).

[18] J. Hamelin, B.R. Gopal, T.K. Bose, J. Thoen, Phys. Rev. Lett. 74, 2733 (1995).

[19] J. Hamelin, T.K. Bose, J. Thoen, Phys. Rev. E 53, 779 (1996).

[20] M. Paluch, P. Hubdas, S.J. Rzoska, T. Schimpel, Chem. Phys. 213, 483 (1996).

[21] J. Goulon, J.L. Greffe, D.W. Oxtoby, J. Chem. Phys. 70, 4742 (1979).

[22] A. Baranowski, M. Dębowska, K. Jerie, A. Jezierski, M. Sachanbinski, Acta Phys. Pol. A 88, 29 (1995).

[23] A. Baranowski, M. Dębowska, K. Jerie, G. Mirkiewicz, J. Rudzińska-Girulska, R.T. Sikorski, J. Phys. IV (France) 3, 225 (1993).

[24] A. Baranowski, K. Jerie, J. Gliński, K. Orzechowski, Acta Phys. Pol. A 90, 1169 (1996).

[25] A. Baranowski, K. Jerie, J. Gliński, J. Radioanal. Nuclear Chem. 211, 3 (1996).

[26] A. Baranowski, K. Jerie, J. Gliński, Chem. Phys. 214, 143 (1997).

[27] K. Jerie, A. Baranowski, B. Rozenfeld, K. Orzechowski, J. Gliński, Chem. Phys. Lett. 137, 42 (1987).

[28] G. Jaci, M.L. Savio, Fast-Slow Coincidence System, Centro Siciliano di Fisica Nucleare e di Struttura della Materia, Catania 1968.

[29] R. Myllylä, On the Measurement Technique of Positron Lifetimes, University of Oulu, Oulu 1976.

[30] K. Jerie, A. Baranowski, B. Rozenfeld, S. Ernst, J. Gliński, Acta Phys. Pol. A 64, 77 (1983). 